\title{
Current Evidence on Exercise for Gestational Diabetes Mellitus in Overweight and Obese Pregnant Women: A Systematic Review
}

\author{
Jialin Li $^{1}$, Zhenglun Yang1, Shuaijun Ren ${ }^{1,2}$, Yajie Gao ${ }^{1,2}$, Yang Song ${ }^{3,4,5}$ and Rongrong Xuan ${ }^{1,2 *}$ \\ ${ }^{1}$ Medical School of Ningbo University, Ningbo University, China \\ ${ }^{2}$ The Affiliated Hospital of Medical School, Ningbo University, China \\ ${ }^{3}$ Faculty of Sports Science, Ningbo University, China \\ ${ }^{4}$ Doctoral School of Safety and Security Sciences, Obuda University, Hungary \\ ${ }^{5}$ Faculty of Engineering, University of Szeged, Hungary
}

*Corresponding author: Rongrong Xuan, Medical School of Ningbo University, Ningbo University, Ningbo, China. The Affiliated Hospital of Medical School, Ningbo University, Ningbo, China.

To Cite This Article: Shuaijun Ren, Yajie Gao, Yang Song, Rongrong Xuan, Current Evidence on Exercise for Gestational Diabetes Mellitus in Overweight and Obese Pregnant Women: A Systematic Review. Am J Biomed Sci \& Res. 2021 - 11(6). AJBSR.MS.ID.001693.

DOI: 10.34297/AJBSR.2021.11.001693

Received: 眥 January 08, 2020; Published: 眥 February 09, 2021

\begin{abstract}
The main purpose of this systematic review was to summarize the recent randomized controlled trials (RCTs) regarding the effects of exercise on gestational diabetes mellitus (GDM) in overweight and obese pregnant women. A literature search was performed on Web of Science, ScienceDirect, and Google Scholar. The keywords were "exercise", "physical activity", "overweight", "obese", "gestational diabetes mellitus" and "gestational diabetes". Only RCTs related to this field that have been published in peer-reviewed journal were covered. Twelve related studies were included in this review, 5 trials employed comprehensive lifestyle programs, 4 trials employed comprehensive exercise training programs, and the remaining 3 trials employed single exercise training programs, respectively. Although there are some conflict results in the effects of exercise on GDM in overweight or obese pregnant women, it seems that single exercise training programs (i.e. moderate-intensity cycling intervention) may contribute to more benefit on reducing the incidence of GDM and improving glucose metabolism. However, further rigorously well-designed RCTs are much warranted for examining the appropriate setting of this intervention and for further verification.
\end{abstract}

Keywords: Exercise; Gestational Diabetes Mellitus; Overweight; Obese; Pregnancy

Abbreviations: RCTs: Randomized Controlled Trials; GDM: Gestational Diabetes Mellitus; T2D: Type-2 Diabetes; BMI: Body Mass Index; N: Number; NA: Not Available; CG: Control Group; EG: Exercise Group; PA: Physical Activity; HE: Healthy Eating

\section{Introduction}

Gestational diabetes mellitus (GDM), similar to type-2 diabetes (T2D), is one of the most common complications of pregnancy, especially for these overweight or obese women [1-3]. It is featured by the disorder of glucose metabolism and carbohydrate [1]. Previous studies have indicated that the prevalence of GDM is increasing greatly worldwide, with the estimated prevalence risk being $17.8 \%$ [3-5]. GDM is associated with many adverse health outcomes both in pregnant women and their infants. For example, compared with healthy counterparts, pregnant women with GDM are more likely to undergo preterm birth, cesarean section, and polyhydramnios [6-9]. In addition, it has been found out that both pregnant women with GDM and their offspring have much higher 
risk of developing T2D and its complications in the future [10]. Therefore, it is of great clinical importance to explore some easy interventions that can be used during pregnancy to alleviate and even cure GDM.

In recent years, a number of studies have indicated that exercise has several benefits on women with GDM [11-15]. For example, Garnæs et al. [14] found that both the incidence of gestational diabetes mellitus and systolic blood pressure were significantly lower after exercise intervention than the control. Several systematic review and meta-analysis on this topic add more evidence. Ming et al. [3] demonstrated that exercise can decrease the incidence of GDM without any adverse results on normalweight pregnant women.

Several factors are highly associated with the occurrence and development of GDM, with overweight or obese being one of the most common one $[16,17]$. However, there is still less evidence based on the systematic evaluation of the effects of exercise on GDM in overweight and obese pregnant women up to now. In addition, emerging studies have revealed that exercise may not benefit overweight pregnant women with GDM $[18,19]$. Therefore, the purpose of this study was to review and summarize the recent randomized controlled trials (RCTs) regarding the effects of exercise on gestational diabetes mellitus (GDM) in overweight and obese pregnant women.

\section{Methods}

\section{Eligibility Criteria}

In order to be included in this review, previous related studies must meet the following several eligibility criteria: 1) study types, only randomized controlled trials (RCTs) that have been published in peer-reviewed English journal and with the aim to investigate the effects of exercise on gestational diabetes mellitus in overweight and/or obese pregnant women were considered for inclusion, other types of research, such as cross-sectional studies, case reports, and thesis etc., were not covered; 2) participants, pregnant women who have been diagnosed as overweight and/or obese based on body mass index (BMI) were included, and there is no restriction on ethnicity and age; 3 ) intervention, participants in the intervention group should perform exercise or take part in some comprehensive exercise programs, while this is no restriction on control group; 4) outcomes, studies evaluated the effects of exercise on parameters related to gestational diabetes mellitus were included.

\section{Searching Strategy}

In order to examine the current evidence regarding this topic, a thorough literature search was applied to identify all related studies from 2010 until 13 October 2020 from the following three English electronic databases: Google Scholar, Web of Science, ScienceDirect. The following several keywords were used during search, ("exercise" OR "physical activity") AND ("overweight" OR "obese") AND ("gestational diabetes mellitus" OR "gestational diabetes"). The searching strategy was modified according to the requirement of each database. In addition, in order to ensure that the searching process is thorough and rigorous, all the retrieved records were independently assessed by two researchers and discussed with the third researcher if any disagreements happened. The citation snowballing method was also applied to check the reference lists of all included studies and locate any potential related studies [20,21].

\section{Data Management}

Two researchers independently extracted and summarized the following study characteristic from each trial, authors, country (where the trial was carried out), published year, participant characteristics (sample size, age, and BMI), interventions (exercise types, frequency, and duration), outcome parameters, and primary results. Any differences that happened during data management would be discussed and resolved with the third researchers. In addition, Mendeley Reference Management Software (Elsevier Ltd., Amsterdam, Netherlands) was used for further study management.

\section{Risk of Bias Assessment}

The Cochrane Risk of Bias Assessment Tool was used to evaluate the quality of all the included studies [22]. To be more specified, seven sub-domains including random sequence generation, allocation concealment, blinding of participants and personnel, blinding of outcome assessment, incomplete outcome data, selective reporting, and other biases, was assessed, and each sub-domain was graded as three levels, low risk of bias, unclear risk of bias, and high risk of bias.

\section{Results}

\section{Searching Results}

The searching and study selecting process is showed in Figure 1. A total of 21,602 records were retrieved from three literature databases, and 452 studies remained after all the irrelevant or duplicate items were removed based on titles. According to the eligibility criteria, 437 papers were then further excluded from this review because of the following several reasons, 1) not randomized controlled trials; 2) studies not related to overweight and/or obese pregnant women; 3) study not involved in exercise intervention; 4) study protocols without any outcomes. After that, 5 duplicates between databases were excluded while 2 additional articles were included after reference searching of the included studies and retrieved reviews. Finally, 12 related studies met the criteria were included in this review.

\section{Study Quality}

The study quality was evaluated in terms of the risk of bias, and the detailed information was presented in Figure 2 \& Table 1. More 
than half of the included studies described the random sequence generation process in detail $(n=9,75.00 \%)$ and concealed the allocation from both participants and researchers $(n=8,66.67 \%)$. However, there is still some studies that did not presented enough detailed information regarding these two domains. Regarding the following two domains, all studies presented high risk of bias

Table 1: Risk of bias summary.

\begin{tabular}{|c|c|c|c|c|c|c|c|}
\hline Trials & $\begin{array}{l}\text { Random } \\
\text { Sequence Gen- } \\
\text { eration }\end{array}$ & $\begin{array}{c}\text { Allocation } \\
\text { Conceal- } \\
\text { ment }\end{array}$ & $\begin{array}{l}\text { Blinding of Par- } \\
\text { ticipants and } \\
\text { Personnel }\end{array}$ & $\begin{array}{l}\text { Blinding of Outcome } \\
\text { Assessment }\end{array}$ & $\begin{array}{l}\text { Incomplete } \\
\text { Outcome Data }\end{array}$ & $\begin{array}{l}\text { Selective Re- } \\
\text { porting }\end{array}$ & $\begin{array}{l}\text { Other } \\
\text { Bias }\end{array}$ \\
\hline Daly et al. (2017) [18] & Low & Low & High & High & Low & Low & Unclear \\
\hline $\begin{array}{l}\text { Hawkins et al. (2015) } \\
{[19]}\end{array}$ & Low & Low & High & High & Low & Low & Unclear \\
\hline Wang et al. (2017) [11] & Low & High & High & High & Low & Low & Unclear \\
\hline Bruno et al. (2017) [12] & Low & Low & High & High & Low & Low & Unclear \\
\hline $\begin{array}{l}\text { Simmons et al. (2017) } \\
{[23]}\end{array}$ & Low & Low & High & High & Low & Low & Unclear \\
\hline Wang et al. (2016) [13] & Unclear & Unclear & High & High & Low & Low & Unclear \\
\hline $\begin{array}{c}\text { Seneviratne et al. (2016) } \\
{[24]}\end{array}$ & Low & Low & High & High & Low & Low & Unclear \\
\hline $\begin{array}{c}\text { Garnæs et al. (2016) } \\
\text { [14] }\end{array}$ & Low & Low & High & High & Low & Low & Unclear \\
\hline Vinter et al. (2018) [25] & Low & Low & High & High & Low & Low & Unclear \\
\hline $\begin{array}{l}\text { Oostdam et al. (2012) } \\
{[26]}\end{array}$ & Unclear & Unclear & High & High & Low & Low & Unclear \\
\hline $\begin{array}{c}\text { Callaway et al. (2010) } \\
\text { [27] }\end{array}$ & Unclear & Unclear & High & High & Low & Low & Unclear \\
\hline $\begin{array}{l}\text { Simmons et al. (2015) } \\
{[28]}\end{array}$ & Low & Low & High & High & Low & Low & Unclear \\
\hline
\end{tabular}

Table 2: The basic characteristics of included studies. because it is usually not possible to blind both participants and researchers when performing interventions $(n=12,100.00 \%)$. Nevertheless, all the studies reported low risk of bias in incomplete outcome data and selective reporting ( $n=12,100.00 \%)$. It is unclear if there is any other bias that existed in these studies $(n=12$, $100.00 \%)$.

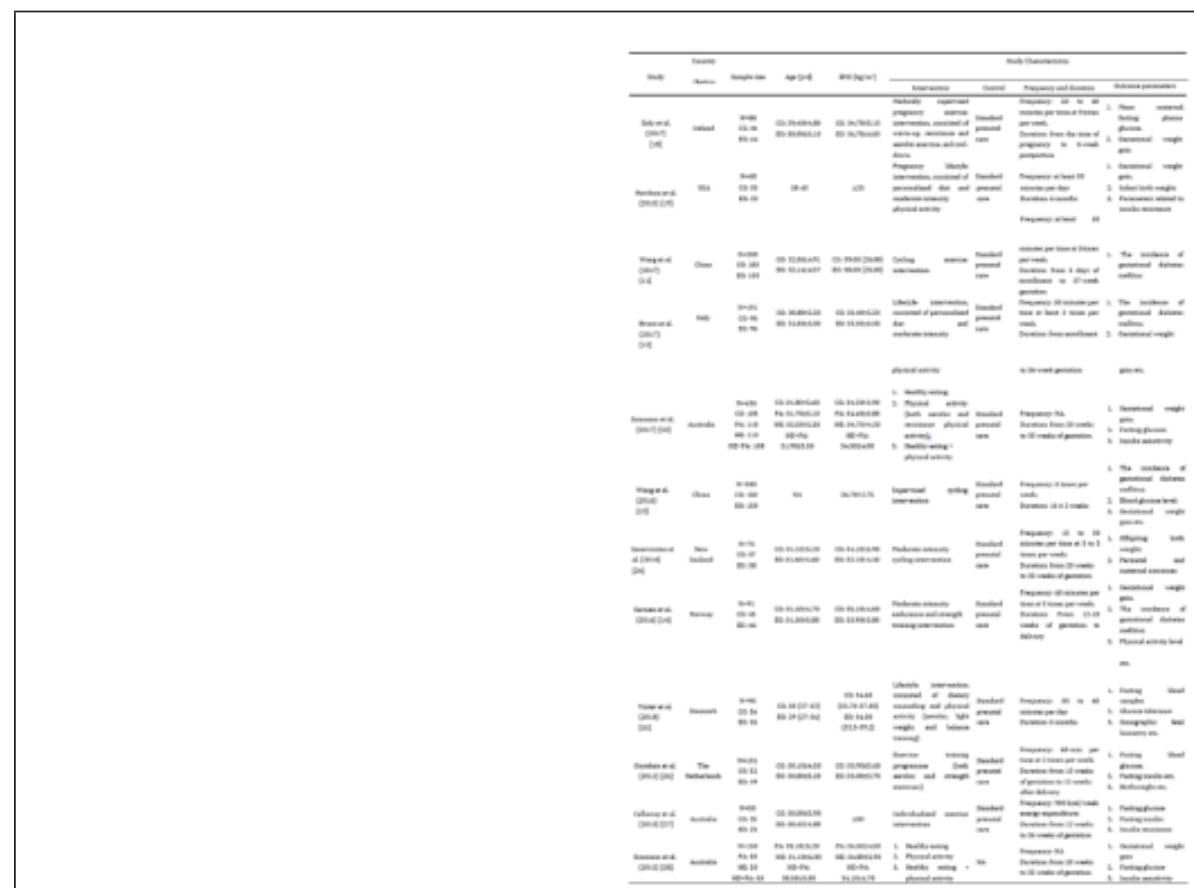


Table 3: The primary results and conclusions of the included studies.

\begin{tabular}{|c|c|}
\hline Trials & Primary results and conclusions \\
\hline Daly et al (2017) [18] & $\begin{array}{l}\text { Although maternal glycemia was not improved after the medically supervised pregnancy exercise intervention, this intervention } \\
\text { significantly reduced the gestational weight gain. }\end{array}$ \\
\hline Hawkins et al. (2015) [19] & > Only the ability for vigorous physical activity was significantly improved after pregnancy lifestyle intervention. \\
\hline Wang et al. (2017) [11] & > Cyeling exereise intervention significantly reduced the incidence of gestational diaberes mellitus. \\
\hline Bruno et al. (2017) [12] & $\begin{array}{l}\text { Lifestyle intervention that including personalized diet and moderate-intensity physical activity significantly reduced the } \\
\text { incidence of gestational diabetes mellitus. }\end{array}$ \\
\hline $\begin{array}{l}\text { Simmons ex al. (2017) } \\
{[23]}\end{array}$ & $\begin{array}{l}\text { Although the gestational weight gain was significantly reduced after healthy eating + physical activity intervention, the fasting } \\
\text { slycemia was not influenced } \\
\text { > None of these three lifestyle interventions can significantily reduce the incidence of gestational diabetes mellitus. }\end{array}$ \\
\hline Wang et al (2016) [13] & $\begin{array}{l}\text { The supervised cycling intervention significantly reduced the incidence of gestational diaberes mellitus and improved glucose } \\
\text { motabolism. }\end{array}$ \\
\hline $\begin{array}{l}\text { Seneviratne et al. } \\
\text { (2016) [24] }\end{array}$ & > Only fitness was significantly improved after the moderate-intensity cycling intervention. \\
\hline Garnxs et al. (2016) [14] & $\begin{array}{l}\text { Although the gestational weight gain was not significantly reduced after moderate-intensity endurance and strength training } \\
\text { intervention, both the incidence of gestational diabetes mellitus and systolic blood pressure seemed to be lower than control } \\
\text { growp. }\end{array}$ \\
\hline Vinter et al (2018) [25] & $\begin{array}{l}\text { Lifestyle intervention that including dietary counseling and physical activity was not effective in improving metabolic and } \\
\text { obstetric parameters. }\end{array}$ \\
\hline Oostdixm et al. (2012) [26] & $>$ No significant effect was observed after exercise training progranme which includes both aevobic and strengthesereises. \\
\hline Callsway et aL. (2010) [27] & $\begin{array}{l}\text { Althought the physical activity ability was improved after individualized exercise intervention, the incidence of gestational } \\
\text { diabotes mellitus was not reduced. }\end{array}$ \\
\hline $\begin{array}{l}\text { Simmons es al. (2015) } \\
{[28]}\end{array}$ & The fasting glucose and gestational weight gain were significantly reduced after healthy eating intervention. \\
\hline
\end{tabular}

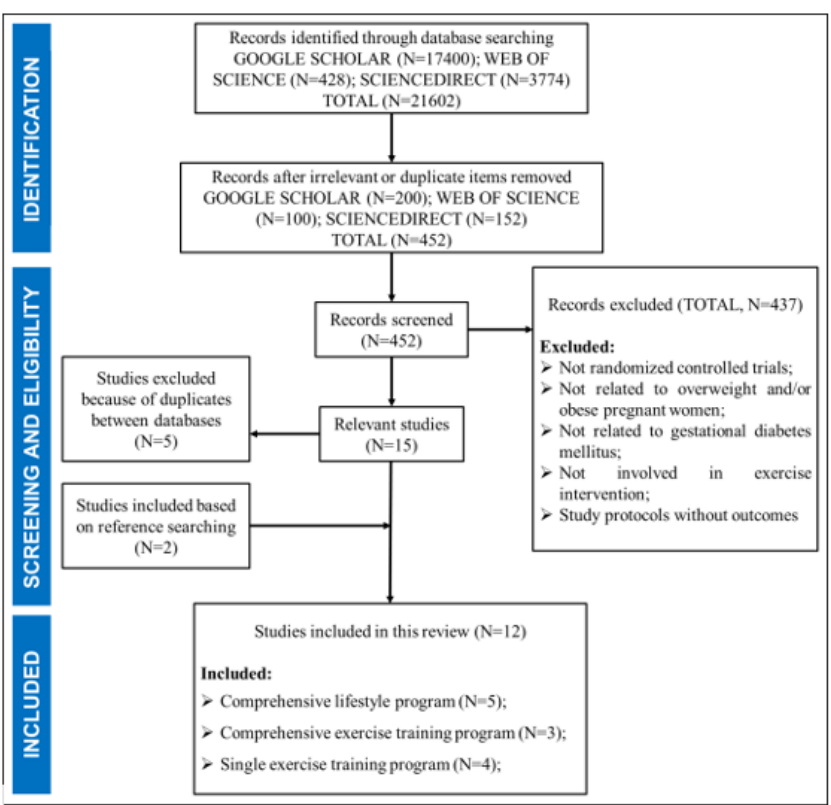

Figure 1: The searching and study selecting flowchart.

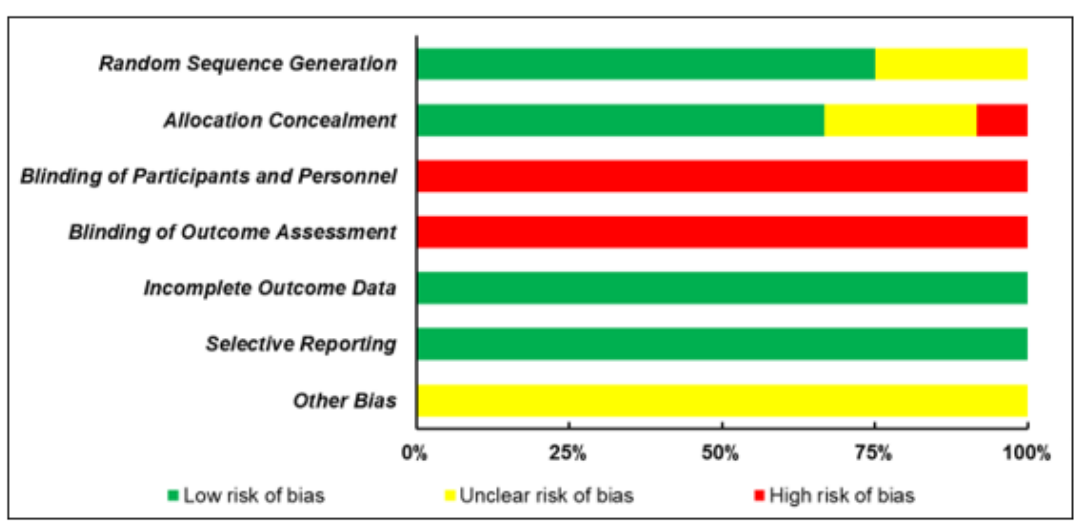

Figure 2: Risk of bias evaluation. 


\section{Basic Study characteristics}

The basic study characteristics were presented in Table 2. Of all the included studies that published in the past 10 years (from 2010 to 2020), most of them were conducted in the Europe (Ireland ( $n=1$ ), Italy $(\mathrm{n}=1)$, Norway $(\mathrm{n}=1)$, Denmark $(\mathrm{n}=1)$, and The Netherlands $(n=1) ; n=5,41.67 \%)$ and Oceania (Australia $(n=3)$ and New Zealand $(n=1) ; n=4,33.33 \%)$. The remaining three studies were conducted in Asia (China, $n=2,16.67 \%$ ) and North America (USA, $n=1,8.33 \%$ ). A total of 1940 pregnant women aged from 18 to 40 years old were covered in these included studies, and all of them were diagnosed as overweight or obese based on their body mass index (BMI). Three kinds of exercise intervention were involved in this review, comprehensive lifestyle program, including both personalized diet and physical activity; comprehensive exercise training program, including different types of exercise (e.g. resistance exercise and aerobic exercise); single exercise training program, including only one type of exercise (cycling). Standard prenatal care was applied in all these studies as the control intervention. The duration of the intervention varied from studies while most studies stated that the intervention was performed from the enrollment day to 3537 weeks of gestation or to delivery. In addition, the frequency of intervention ranged from 2 to 5 times per week, with each time lasts for 15 to 60 minutes.

The primary outcomes of these included studies were presented in Table 3. Summary of these resulted were displayed in 3 parts, including 1) effects of comprehensive lifestyle program on GDM; 2) effects of comprehensive exercise training program on GDM; 3) effects of single exercise training program on GDM.

\section{Effects of Comprehensive Lifestyle Program on GDM}

Most studies $(n=5)$ in this review investigated the effects of comprehensive lifestyle program on GDM.

Two studies related to this topic were conducted in 2015. Hawkins and his colleagues [19] compared the effects of pregnancy lifestyle intervention, which is consisted of personalized diet and moderate-intensity physical activity, with standard prenatal care on pregnant women. Subjects were asked to perform this intervention at least 30 minutes per day for 6 months in total. Gestational weight gain, infant birth weight, and parameters related to insulin resistance were measured before and after intervention, and they found that only the ability for vigorous physical activity was significantly improved when compared to the control group. Although there are some differences in study design, Simmons et al. [28] reported some positive results. Subjects were divided into three groups, healthy eating group, physical activity group, and healthy eating + physical activity group, and were required to perform corresponding intervention from 20 weeks to 35 weeks of gestation. They found that the fasting glucose and gestational weight gain were significantly reduced after healthy eating intervention, but not for the other two groups. However, using the same study design, Simmons et al. [23] presented some different outcomes. They demonstrated that although the gestational weight was significantly reduced after healthy eating + physical activity intervention, none of these three interventions can significantly reduce the incidence of gestational diabetes mellitus.

Finally, two recent studies in this category also reported some controversial results. Bruno et al. [12] compared the effects of pregnancy lifestyle intervention (30 minutes per time for 3 times per week from enrollment to 36-week gestation) with standard prenatal care, and they found that this comprehensive lifestyle program significantly reduced the incidence of GDM. Vinter et al. [25] conducted a similar trial on pregnant women. Subjects were asked to perform comprehensive intervention 30 to 60 minutes per day for 6 months in total, and they reported that lifestyle intervention was not effective in improving metabolic and obstetric parameters.

\section{Effects of Comprehensive Exercise Training Program on GDM}

Four studies evaluated the effects of comprehensive exercise training program on GDM were included in this category. Callaway et al. [27] firstly started RCT on obese pregnant women. Subjects were asked to perform individualized exercise intervention from 12 weeks to 36 weeks of gestation, and they were required to meet the $900 \mathrm{kcal}$ energy expenditure per week. Similar to what Hawkins et al. has found, the incidence of GDM was not reduced although the physical activity ability was improved after individualized exercise intervention. In addition, Oostdam et al. [26] also reached a relatively similar result. A 60-min course of exercise training program including both aerobic and strength exercise was applied in their study at 2 times per week from 15 weeks of gestation to 12 weeks after delivery, and the results shown that no significant effect was observed after exercise training programme when compared to control group. The subsequent two studies also put some conflict results. In Garnæs et al. [14] study, subjects were randomly assigned to a 60-min moderate-intensity endurance and strength training intervention at 3 times per week or standard prenatal care from 12-18 weeks of gestation to delivery, and they found that although the gestational weight gain was not significantly reduced, both the incidence of gestational diabetes mellitus and systolic blood pressure seemed to be lower after moderate-intensity endurance and strength training intervention than control group. However, using the similar study design, Daly et al. [18] demonstrated that the comprehensive exercise training program can significantly reduce the gestational weight gain but have no effect on maternal glycemia. 


\section{Effects of Single Exercise Training Program on GDM}

There are also 3 studies focused on investigating the effects of single exercise training program on GDM. The first of the three studies compared the effects of moderate-intensity cycling intervention with standard prenatal care on GDM [24]. The results showed that only fitness was significantly improved after exercise intervention. However, Wang et al. conducted two trials in 2016 [13] and 2017 [11] and found some different results. Although there are some differences in the setting of exercise intervention, both studies demonstrated that the cycling exercise intervention significantly reduced the incidence of GDM.

\section{Discussion}

This study comprehensively reviewed and summarized evidences from previous RCTs examining the effects of exercise on GDM in overweight or obese pregnant women, with the aim to find out if there are any better exercise interventions that may contribute to more benefits for the treatment of GDM.

Although there are many comprehensive exercise interventions and single exercise training programs that have already been used, conflict results always existed. For the comprehensive lifestyle program, 4 studies reported that it has no positive effects on GDM $[19,23,25,28]$ while 1 study did find some positive influence after intervention [12]. It is interesting to note that Simmons et al. conducted two studies in 2015 [28] and 2017 [23] using the same study design respectively but the results were relatively different. The former study found that the fasting glucose and gestational weight gain were significantly reduced after healthy eating intervention while the latter demonstrated that although the gestational weight was significantly reduced after healthy eating + physical activity intervention, none of these three interventions can significantly reduce the incidence of gestational diabetes mellitus. The reason for this difference could be the number of subjects in these two studies as Simmons et al. [23] increased the subject number to 436 . There is a similar trend for the comprehensive exercise training programs, with 3 neutral results $[18,26,27]$ and 1 positive result [14]. However, there is a relatively different trend for the single exercise training program, with 2 positive results $[11,13]$ and 1 neutral result [24]. To sum up, from the recent evidence, it seems that moderate-intensity cycling intervention may have some positive effects on GDM in overweight or obese women, nevertheless, more rigorously well-designed RCTs are needed for further verification.

Two potential limitations of this review and design flaws of these included studies should be mentioned and addressed in the future studies. Firstly, in order to examine the recent evidence regarding this topic, only studies published in 2010 to 2020 were included in this review, which may be one of the main reasons why this review failed to reach a unified conclusion. Further related reviews should expand the searching range. Secondly, of the 12 included studies, only half studies have a sample size of more than 100 , which may increase the type $\Pi$ error risk of their results. Thus, further studies with larger sample size in this field are much needed.

\section{Conclusions}

In conclusion, although there are some conflict results in the effects of exercise on GDM in overweight or obese pregnant women, it seems that moderate intensity cycling intervention may contribute to more benefit on reducing the incidence of GDM and improving glucose metabolism. However, more rigorously welldesigned RCTs are warranted to examine the appropriate setting and for further verification.

\section{Conflicts of Interest}

The authors declare no conflicts of interest.

\section{References}

1. Buchanan TA, Xiang AH, Page KA (2012) Gestational diabetes mellitus: Risks and management during and after pregnancy. Nat Rev Endocrinol 8(11): 639-649.

2. Carol A Snapp, Sue K Donaldson (2008) Gestational diabetes mellitus: Physical exercise and health outcomes. Biol Res Nurs 10(2): 145-155.

3. Ming WK, Ding W, Zhang CJP, Zhong L, Long Y, et al. (2018) The effect of exercise during pregnancy on gestational diabetes mellitus in normal-weight women: A systematic review and meta-analysis. BMC Pregnancy Childb 18(1): 1-9.

4. Boyd E Metzger, Lynn P Lowe, Alan R Dyer, Elisabeth R Trimble, Udom Chaovarindr, et al. (2008) Hyperglycemia and adverse pregnancy outcomes. N Engl J Med 358(19): 1991-2002.

5. Boyd E Metzger, Bengt Persson, Lynn P Lowe, Alan R Dyer, J Kennedy Cruickshank, et al. (2010) Hyperglycemia and adverse pregnancy outcome study: Neonatal glycemia. Pediatrics 126(6): 1545-1552.

6. Langer O, Mazze R (1988) The relationship between large-for-gestational-age infants and glycaemic control in women with gestational diabetes. Am J Obstet Gynecol 159(6): 1478-1483.

7. Assiamira Ferrara, Henry S Kahn, Charles P Quesenberry, Candice Riley, Monique M Hedderson (2004) An increase in the incidence of gestational diabetes mellitus: Northern California, 1991-2000. Obstet Gynecol 103(3): 526-533.

8. Caroline A Crowther, Janet E Hiller, John R Moss, Andrew J McPhee, William S Jeffries, et al. (2005) Effect of treatment of gestational diabetes mellitus on pregnancy outcomes. N Engl J Med 352(24): 2477-2486.

9. Mark B Landon, Catherine Y Spong, Elizabeth Thom, Marshall W Carpenter, Susan M Ramin, et al. (2009) A multicenter, randomized trial of treatment for mild gestational diabetes. N Engl J Med 361(14): 1339-1348.

10. Bellamy L, Casas JP, Hingorani AD, Williams D (2009) Type 2 diabetes mellitus after gestational diabetes: a systematic review and meta-analysis. Lancet 23,373(9677): 1773-1779.

11. Chen Wang, Yumei Wei, Xiaoming Zhang, Yue Zhang, Qianqian Xu et al. (2017) A randomized clinical trial of exercise during pregnancy to prevent gestational diabetes mellitus and improve pregnancy outcome in overweight and obese pregnant women. Am J Obstet Gynecol 216(4): $340-351$. 
12. Raffaele Bruno, Elisabetta Petrella, Valentina Bertarini, Giulia Pedrielli, Isabella Neri, et al. (2017) Adherence to a lifestyle programme in overweight/obese pregnant women and effect on gestational diabetes mellitus: a randomized controlled trial. Matern Child Nutr 13(3): 12333.

13. Wang C, Wei Y, Zhang X, Zhang Y, Xu Q et al. (2016) Effect of regular exercise commenced in early pregnancy on the incidence of gestational diabetes mellitus in overweight and obese pregnant women: A randomized controlled trial. Diabetes Care 39(10): 163-164.

14. Garnæs KK, Morkved S, Salvesen O, Moholdt T (2016) Exercise training and weight gain in obese pregnant women: A randomized controlled trial (ETIP Trial). PLoS Med 13(7): 1002079.

15. James F Clapp (2006) Effects of diet and exercise on insulin resistance during pregnancy. Metab Syndr Relat Disord 4(2): 84-90.

16. Patrick M Catalano, H David McIntyre, J Kennedy Cruickshank, David R McCance, Alan R Dyer, et al. (2012) The hyperglycemia and adverse pregnancy outcome study: Associations of GDM and obesity with pregnancy outcomes. Diabetes Care 35(4): 780-786

17. Ulla Kampmann, Lene Ring Madsen, Gitte Oeskov Skajaa, Ditte Smed Iversen, Niels Moeller (2015) Gestational diabetes: A clinical update. World J Diabetes 6(8): 1065

18. Niamh Daly, Maria Farren, Aoife McKeating, Ruth O Kelly, Mary Stapleton, et al. (2017) A medically supervised pregnancy exercise intervention in obese women: A randomized controlled trial. Obstet Gynecol 130(5): 1001-1010.

19. Hawkins M, Hosker M, Marcus BH, Rosal MC, Braun B, et al. (2015) A pregnancy lifestyle intervention to prevent gestational diabetes risk factors in overweight Hispanic women: A feasibility randomized controlled trial. Diabet Med 32(1): 108-115.

20. Song Y, Ren F, Sun D, Wang M, Baker JS, et al. (2020) Benefits of exercise on influenza or pneumonia in older adults: A systematic review. Int J Environ Res Public Health 13,17(8): 2655.
21. Song Y, Sun D, Istvan B, Thirupathi A, Liang M, et al. (2020) Current evidence on traditional chinese exercise for cancers: A systematic review of randomized controlled trials. Int J Environ Res Public Health 17(14): 5011.

22. Higgins JPT, Altman DG, Gotzsche PC, Jüni P, Moher D, et al. (2011) The Cochrane Collaboration's tool for assessing risk of bias in randomised trials. BMJ 18(343): 5928

23. Simmons D, Devlieger R, Van Assche A, Jans G, Galjaard S, et al. (2017) Effect of physical activity and/or healthy eating ongdm risk: The dali lifestyle study. J Clin Endocrinol Metab 102(3): 903-913.

24. Seneviratne SN, Jiang Y, Derraik JGB, McCowan LME, Parry GK, et al. (2016) Effects of antenatal exercise in overweight and obese pregnant women on maternal and perinatal outcomes: A randomised controlled trial. BJOG: An Int J Obstet Gynaecol 123(4): 588-597.

25. Vinter CA, Tanvig MH, Christensen MH, Ovesen PG, Jorgensen JS, et al. (2018) Lifestyle intervention in Danish obese pregnant women with early gestational diabetes mellitus according to WHO 2013 criteria does not change pregnancy outcomes: Results from the LIP (Lifestyle in Pregnancy) study. Diabetes Care 41(10): 2079-2085.

26. Oostdam N, Van Poppel MNM, Wouters MGAJ, Eekhoff EMW, Bekedam DJ, et al. (2012) No effect of the FitFor2 exercise programme on blood glucose, insulin sensitivity, and birthweight in pregnant women who were overweight and at risk for gestational diabetes: Results of a randomised controlled trial. BJOG: An Int J Obstet Gynaecol 119(9): 1098-1107.

27. Callaway LK, Colditz PB, Byrne NM, Lingwood BE, Rowlands IJ, et al. (2010) Prevention of gestational diabetes: Feasibility issues for an exercise intervention in obese pregnant women. Diabetes Care 33(7): 1457-1459.

28. Simmons D, Jelsma JGM, Galjaard S, Devlieger R, Van Assche A, et al (2015) Results from a European multicentre randomized trial of physical activity and/or healthy eating to reduce the risk of gestational diabetes mellitus: The DALI lifestyle pilot. Diabetes Care 38(9): 1650-1656. 